\title{
ABASTECIMIENTO DE PRODUCTOS BÁSICOS A ZACATECAS DURANTE LOS SIGLOS XVI Y XVII
}

\author{
THE SUPPLY OF COMMODITIES TO ZACATECAS \\ DURING THE XVITH AND XVIITH CENTURIES
}

\author{
Ana Guillermina Gómez Murillo \\ Universidad de Guadalajara \\ Centro Universitario de Ciencias Sociales y Humanidades \\ anaguille_82@yahoo.com.mx \\ ORCID: 0000-0002-9741-4909
}

\section{Resumen}

Este artículo trata sobre los desafíos que conllevó el abasto de productos básicos a Zacatecas. Específicamente explica la conformación de regiones abastecedoras de granos, productos básicos y la organización del comercio en este real de minas novohispano que marcaría las nuevas rutas exploratorias de los peninsulares al septentrión y un nuevo derrotero económico con la consolidación de la minería como eje de la economía novohispana. Una pieza fundamental en la regularización del comercio fue el cabildo de la ciudad.

Palabras clave: comercio; abasto; minería; ciudades novohispanas; ganadería.

\begin{abstract}
This article explains the challenges that arose with the supply of the basic products brought to Zacatecas, more specifically, the establishment of grain supplying regions, commodities, and the organization of trade in this Spanish Colonial mining town. It would indicate the beginning of the exploratory routes that took the peninsular Spaniards to the northern and how it led to a new economic direction with the consolidation of mining as the base of New Spain economy. A fundamental piece in the regularization of commerce was the town council.
\end{abstract}

Keywords: trade; supply; mining; New Spain cities; cattle raising.

\section{Información del artículo}

Recibido: 3 de julio de 2019.

Aceptado: 21 de abril de 2020.

DOI: http://dx.doi.org/10.22201/iih.24486922e.2020.63.70192 
...y como el sitio en que se descubren las minas es infructífero de los necesarios mantenimientos, logran los labradores y criaderos de los contornos el espendio de sus semillas y ganados; y como éstos solos no pueden dar abasto al gentío que concurre, se ven precisados otros, o por la necesidad o por la codicia, a descubrir nuevas labores y poblar nuevas estancias de ganados, aun las tierras de mayor peligro por los bárbaros, disponiendo Dios por este medio, que aunque las minas decrezcan, queden las tierras circunvecinas con las nueva labores y estancias bien pobladas y con suficiente comercio entre sus moradores.

José De ArLegui (1737)*

El presente texto se enfoca en el abasto de Zacatecas en su etapa inicial. La investigación se realizó principalmente a través del seguimiento de actas de la Diputación de Minas y posteriormente del Cabildo de la ciudad, así como algunos contratos de abastecimiento de carne, entre otros documentos. Se hace patente el papel articulador que una ciudad minera como Zacatecas tuvo para el comercio de algunas mercancías de distintas regiones novohispanas durante el periodo señalado, al tiempo que se conformaron áreas cercanas de aprovisionamiento de productos básicos.

Al hacer el seguimiento documental se establece que el área de abasto de granos se extendió hacia el norte de Jalisco y sur de Zacatecas, así como a Sombrerete y áreas circunvecinas como el Valle de Súchil en Durango. Por su parte, el abasto de cárnicos y productos derivados de la ganadería se dio en las inmediaciones de los centros mineros debido a la proliferación de estancias ganaderas. El abasto de productos de origen europeo dependió del comercio interoceánico. En el caso del vino, existió un activo comercio ilegal y la introducción de vino de cocos que provenía de la costa del Pacífico. Por último, se hace referencia a insumos madereros, ya que fueron fundamentales para la actividad minera. La transformación del paisaje debido a la actividad extractiva conllevó que se dictaran algunas normativas en favor de su administración.

* José de Arlegui, Crónica de la Provincia de San Francisco de Zacatecas (México: José Bernardo de Hogal, 1737), 122. 
Un enclave minero

El descubrimiento e inicio del poblamiento de Zacatecas ocurrió a partir de 1546; sin embargo, para el año de 1612 se convirtió en la tercera ciudad novohispana en importancia. ${ }^{1} \mathrm{El}$ auge se dio ante condiciones adversas y se debió a la colaboración de mineros que invirtieron de forma directa y por medio de asociaciones. También las concesiones fiscales y las medidas que favorecieron a la región por parte de la Corona auxiliaron en esta consolidación. ${ }^{2}$

Hacia 1548 se localizaron los tres grupos principales de vetas que dieron un auge inicial, las cuales estaban siendo explotadas por personajes que fueron trascendentales para el desarrollo de la minería en la región: Miguel de Ibarra y Juan de Tolosa. En 1549 ya había trescientas personas en el real y los mineros enviaron a la Audiencia de Compostela, como procurador, a Juan de Zaldívar, con la solicitud de la creación de la alcaldía mayor. En respuesta a ello, se dio la visita del oidor Hernán Martínez de la Marcha en 1550. A partir de entonces se dictaron diversas ordenanzas para el establecimiento del orden en las actividades productivas y la organización de los habitantes. Lacueva Muñoz atribuye, de cierto modo, a estas normativas el primer auge que experimentó la minería en la región, ya que gracias a ellas se contó con parámetros para la regulación de la extracción, así como la resolución de conflictos que se suscitaban entre los mineros. ${ }^{3}$

En 1553 se estableció la diputación de minas, forma de organización que posteriormente derivó en el cabildo de la ciudad y más tarde, en 1562, se dictaron las ordenanzas para la cosecha de sal en las Salinas Grandes por parte del oidor Francisco Gómez de Mendiola. Posteriormente, en 1568, el mencionado Gómez de Mendiola expió las ordenanzas concernientes al aprovechamiento de los recursos forestales, y otras fueron dictadas en 1576 tras la visita del oidor Santiago del Riego. ${ }^{4}$

${ }^{1}$ Jaime J. Lacueva Muñoz, “Zacatecas: norte imperial”, en Historia del Reino de la Nueva Galicia, coord. de Thomas Calvo y Aristarco Regalado Pinedo (Guadalajara: Universidad de Guadalajara, 2016), 537.

${ }^{2}$ Lacueva Muñoz detalla extensamente los diversos procesos que confluyeron en la consolidación de Zacatecas como real de minas y punto de exploración hacia el norte novohispano. Jaime Lacueva Muñoz, La plata del rey y sus vasallos. Minería y metalurgia en México (siglos XVI $y_{X V I I}$ ) (Sevilla: Consejo Superior de Investigaciones Científicas, Escuela de Estudios HispanoAmericanos; Sevilla: Universidad de Sevilla; Sevilla: Diputación de Sevilla, 2010).

${ }^{3}$ Lacueva, "Zacatecas", 538-540.

${ }^{4}$ Lacueva, "Zacatecas", 539. 
La regulación de distintas actividades se había llevado a cabo por medio de ordenanzas en la península ibérica desde el siglo xiı de forma consistente. Este tipo de instrumentos tuvo como objetivo lograr una mejor convivencia y normar las actividades en una sociedad estamental. Por lo anterior, las actividades realizadas por los novohispanos fueron reguladas mediante ordenanzas durante el periodo virreinal. ${ }^{5}$ Entre 1550 y 1555 se puso en servicio el Camino Real de Tierra Adentro gracias a los empeños de los virreyes Mendoza y Velasco el Viejo. ${ }^{6}$ La seguridad de esta importante vía de comunicación se vio fortalecida con fundaciones que salvaguardaron la ruta, y que de igual forma significaron el aprovisionamiento de bienes agrícolas como Celaya, León, Aguascalientes, Jerez y Ojuelos. ${ }^{7}$

En 1553 se concedió la creación de la Diputación de Minas. El principal objetivo era lograr un contrapeso comercial que auxiliara en tasaciones de precios más benéficos para los mineros y la población flotante. Sin embargo, lo anterior no se logró del todo, debido a la escasa competencia comercial por la lejanía de Zacatecas.

A partir de 1548, los mineros comenzaron a solicitar rebajas de impuestos; en esa ocasión se concedió la reducción de quinto a diezmo, y posteriormente otorgaron una prórroga. En la coyuntura de la inseguridad de los caminos, los mineros solicitaron una rebaja en 1556 , la cual también fue autorizada. Por último, en 1559 lograron la disminución de diezmo a veinteno. ${ }^{8}$

Durante los años posteriores al inicio de la extracción de mineral en Zacatecas, se dio una fuerte oleada exploratoria. El proveer de insumos básicos a estos reales fue una tarea difícil; de ahí la urgencia de fundar zonas de producción de insumos agrícolas y ganaderos. ${ }^{9}$

La diputación de minas y el cabildo hicieron importantes esfuerzos de regularización del comercio y de precios. Los primeros miembros de estas instituciones en su etapa inicial eran mineros. Se aprecia que hubo una rotación en sus miembros para las decisiones comerciales, y pocos fueron los que se repetían, a excepción de algunos pertenecientes a la élite primaria,

5 Thomas Calvo, "Ordenanzas municipales para el buen gobierno”, en Horizontes de monarquía. Siete estudios de caso desde el "Potosí" mexicano, coord. de Óscar Mazín Gómez y Armando Hernández Soubervielle (México: El Colegio de San Luis, 2018), 51-94.

${ }^{6}$ Lacueva, “Zacatecas”, 540.

7 Lacueva, "Zacatecas", 553.

${ }^{8}$ Lacueva, “Zacatecas”, 541-543.

9 Lacueva, “Zacatecas”, 544. 
como fueron Vicente y Cristóbal de Zaldívar o Diego Temiño de Bañuelos, quienes pertenecieron a la élite principal de la ciudad (véase el cuadro 1). ${ }^{10}$

Productos básicos para un nuevo real de minas

Desde el descubrimiento de las minas de Zacatecas en el siglo XVI, se fueron conformando zonas aledañas especializadas en agricultura y ganadería, aunque los grandes productores de granos estaban asentados en el Bajío y Michoacán. Para ello, se creó una red eficiente de transporte en recuas de mulas y carros jalados por bueyes que fueron capaces de llevar todas las mercancías y frutos del campo a través de las rutas principales. ${ }^{11} \mathrm{El}$ enlace de zonas productoras de grano del norte de Michoacán con el camino real es un ejemplo de la fuerte dependencia que tenía el distrito minero de Zacatecas para la obtención de productos básicos. ${ }^{12}$

Para transportar la plata a la ciudad de México y llevar los bastimentos a la ciudad de Zacatecas se desarrolló una extensa red de caminos, incluido el camino real de las minas de Zacatecas, por donde transitaban carros y recuas. A lo largo de estos caminos se fundaron presidios, guarniciones, fuertes y ventas, que con el tiempo se trasformaron en importantes pueblos y villas. ${ }^{13}$

La alhóndiga se estableció en el siglo xviı y los controles de precios no fueron del todo efectivos en algunos productos. Por lo anterior, el peligro que conllevaba el transporte de mercancías durante las primeras décadas de la explotación argentífera se compensaba con las ganancias que pudieran obtenerse. ${ }^{14}$ Se hacían alrededor de cuatro semanas para recorrer los caminos México-Guadalajara; de tres a cuatro para el de Guadalajara-Zacatecas

10 Thomas Hillerkuss, "Una sociedad en construcción. La organización de la élite minera en Zacatecas durante el siglo xvı", en México a la luz de sus revoluciones, coord. de Laura Rojas y Susan Deeds (México: El Colegio de México, 2017), 93-96.

11 Thomas Calvo, Por los caminos de Nueva Galicia. Transportes y transportistas en el siglo XVII (México: Universidad de Guadalajara; México: Centro Francés de Estudios Mexicanos y Centroamericanos, 1997), 21.

${ }^{12}$ Peter J. Bakewell, Minería y sociedad en el México colonial. Zacatecas 1546-1700 (México: Fondo de Cultura Económica, 1971), 87.

${ }^{13}$ Jesús Flores Olague, Mercedes de Vega et al., Breve historia de Zacatecas (México: El Colegio de México; México: Fondo de Cultura Económica, 1996), 76.

${ }^{14}$ Bakewell, Minería, 87. 
Cuadro 1

Miembros DE LA DIPUTACión DE MINAS Y CABILDO DE ZACATECAS

Año Miembros

Alcalde mayor: Rodrigo Sánchez.

1575 Rector: Alonso Gutiérrez del Campo.

Diputados: Vicente de Zaldívar, Cristóbal de Argüello y Alonso Fernández.

Alcalde mayor: Rodrigo Sánchez.

1576 Rector: Cristóbal de Argüello.

Diputados: Gaspar de Ortega, Juan de Rentería y Antonio Salas.

Corregidor: Félix de Zúñiga y Avellaneda.

1584 Rector: Vicente de Zaldívar.

Diputados: Alonso Fernández Bachiller, Antonio de Salas y Ruy García de Ortega.

Corregidor: licenciado Juan Núñez.

1588

Alcaldes ordinarios: Vicente de Zaldívar y Baltasar de Bañuelos.

Regidor y procurador general: Cristóbal de Zaldívar.

Regidores: Bautista de Salas, Hernando de Burgos, Pedro Venegas, Juan Delgado.

Corregidor: licenciado Juan Núñez.

1590 Alcaldes ordinarios: Cristóbal de Zaldívar y Mateo del Río.

Regidor y procurador general: Antonio López de Cepeda.

Regidores: Alonso Sánchez, Pedro Venegas, Sebastián Muñoz.

Corregidor: Diego Fernández de Velasco.

1593 Alcaldes ordinarios: Alonso Sánchez y Mateo del Río.

Regidores: Alonso de Ávila León y Juan Marmolejo Tinoco.

Corregidor: Antonio de Saavedra.

1594 Alcaldes ordinarios: Antonio López de Cepeda.

Regidor: Antonio de Figueroa.

Corregidor: Antonio de Saavedra.

1596 Acaldes ordinarios: Francisco Gutiérrez Trejo y Sebastián Muñoz.

Regidor: Cristóbal Martínez. 


\begin{tabular}{ll}
\hline Año & Miembros \\
\hline
\end{tabular}

Teniente de corregidor: Nicolás de Rodríguez.

1597 Alcaldes ordinarios: Diego de los Ríos Proaño y Ruy García de Ortega. Regidor: Cristóbal Martínez.

Corregidor: Fernando de Valdés.

1600 Alcaldes ordinarios: Nicolás Rodríguez y Juan de Monroy.

Regidor: Cristóbal Martínez.

Corregidor: Fernando de Valdés.

1603 Alcaldes ordinarios: Cristóbal de Zaldívar y Juan Cortés.

Regidor: Cristóbal Martínez.

Corregidor: Juan de Guzmán.

1608 Alcaldes ordinarios: Pedro de Alcaraz y Juan Yáñez.

Regidor: Cristóbal Martínez.

Corregidor: Francisco Juárez de Ezpeleta.

1615 Alcaldes ordinarios: Diego Temiño de Bañuelos y Diego Pérez de Rivera.

Regidores: Cristóbal Martínez y Pedro de Ortega.

Corregidor: Antonio de Figueroa.

1622 Alcaldes ordinarios: Francisco de Castro y Juan Yáñez.

Regidor: Cristóbal Martínez, Juan de Monroy y Pedro de Ortega.

Corregidor: Diego de Medrano.

1623 Alcaldes ordinarios: Agustín de Zavala y Vicente de Zaldívar Mendoza.

Regidores: Pedro de Ortega y Pedro de Enciso.

Corregidor: Francisco Hernández Zapata.

1635 Alcaldes mayores: Lorenzo Ruiz Tostado y Juan Ramírez Ponce.

Regidores: Pedro de Enciso, Martín Sánchez de Vera y Pedro Serrano.

Fuente: AHez, Ayuntamiento, Actas de Cabildo, libros 1-3. 
y hasta seis semanas para el de México-Zacatecas. Este transporte, efectivo pero lento, impedía la estabilidad de los precios, los cuales arreciaban en años de escasez de lluvias o de malas cosechas por heladas tardías o tempranas. Durante los siglos XVI y XVII, una parte importante de la producción de la Nueva Galicia estuvo destinada a Zacatecas.

Al descubrimiento de Zacatecas lo siguieron otros reales con sus respectivos caminos e inicios del abasto de productos básicos:

El camino Zacatecas-Durango se convirtió rápidamente en una vía muy transitada; la más importante, de hecho, al norte de la Nueva Galicia. Fue éste un tramo bastante poblado desde épocas muy tempranas. Resguardaba la ruta, los poblados de Fresnillo (real descubierto en 1563); el Saín, que se pobló pronto de españoles; Sombrerete, San Martín y Nombre de Dios, además de otras haciendas agrícolas, de tal manera que los viajeros encontraban fácilmente dónde descansar y abastecerse. A dos años apenas de haberse fundado la Nueva Vizcaya, ningún punto poblado del camino entre Zacatecas y Durango se encontraba a más de $40 \mathrm{~km}$ del siguiente. Se había creado una importante extensión del Camino Real de Tierra Adentro: la ruta Zacatecas-Durango. ${ }^{15}$

Factores como la inseguridad retrasaron el proceso de consolidación de esas zonas productoras. Previamente a la conformación de las haciendas, se estableció una serie de presidios, los cuales irían acompañados de poblaciones a fin de asegurar el avance hacia el norte. "El presidio reunió y protegió a la población pero - además- creó y reforzó la producción en vastas zonas agrícolas y ganaderas, de ahí la formación necesaria y natural de un aparato defensivo entre esos centros de producción y los de abasto". ${ }^{16}$ En los siglos posteriores, estos presidios fueron desapareciendo; algunos se transformaron en haciendas de campo, pueblos o villas. Así se consolidaron los poblados de Fresnillo, Aguascalientes, Ojuelos, Malpaso, Tlaltenango, Sombrerete, Colotlán, villa de Santa María de los Lagos y Chalchihuites.

Entre las zonas agrícolas articuladas al distrito minero de Zacatecas, en el siglo XVI, encontramos a Jerez, que fue la zona de provisionamiento de granos más cercana al real de minas junto con otros poblados de Aguascalientes,

${ }^{15}$ Chantal Cramaussel, "El Camino Real de Tierra Adentro. De México a Santa Fe", en Rutas de la Nueva España, ed. de Chantal Cramaussel (Zamora, Michoacán: El Colegio de Michoacán, 2006), 303.

${ }^{16}$ José Manuel Martín Ornelas, “La organización económica regional y el abasto urbano. El trigo y el maíz en Zacatecas. 1749-1821" (tesis para obtener el grado de doctor en Humanidades, Universidad Autónoma de Zacatecas, Zacatecas, 2008), 76. 
sur de Zacatecas y norte de Jalisco. La población de esta zona estuvo compuesta principalmente por labradores y conservó durante el resto del periodo virreinal su especialización agrícola.

Otros lugares de relevancia cerealera fueron Fresnillo, donde se cultivaba maíz y trigo, y la zona de Los Cañones, que tuvo también una producción significativa de grano desde épocas tempranas, como el caso de Tlaltenango. Por su parte, Sombrerete se abasteció básicamente del valle del Súchil y de Poanas, en la Nueva Vizcaya.

Hacia 1585 ya se habían aclimatado diversos cultivos indígenas y europeos en los reales de minas descubiertos, como árboles frutales, calabaza, chile y tomate, así como trigo, cebada, garbanzos y lentejas, los cuales se sembraban para autosubsistencia. ${ }^{17}$

Entre otras importaciones de productos básicos se encontraba el azúcar, que era traída desde la tierra caliente de Michoacán, así como los embutidos, que pronto fueron elaborados en la zona de Teocaltiche y en Los Cañones. Las hortalizas y frutas eran sembradas principalmente en las poblaciones mismas y en sus inmediaciones o en pueblos de indios, gracias a la existencia de numerosos huertos, ${ }^{18}$ situación que se repitió en casi todos los lugares visitados por el obispo Alonso de la Mota y Escobar, ${ }^{19}$ y en las Relaciones geográficas del siglo XVI.

Durante los siglos XVI y XVII se hizo una importante adquisición de tierras por parte de prominentes mineros, como Diego de Ibarra, Baltasar Temiño de Bañuelos, Juan Guerra de Reza, Alonso López de Lois, Rodrigo del Río de Loza, Juan Bautista Lomas de Colmenares y Francisco de Urdiñola; éstos destinaron sus propiedades a la agricultura y la ganadería, ya fuera para autoabastecer sus explotaciones mineras o como ingresos

17 René Acuña, ed., Relaciones geográficas del siglo XVI. Nueva Galicia (México: Universidad Nacional Autónoma de México, 1988), 117-123.

${ }^{18}$ Los huertos son componentes de sistemas agrícolas donde las plantas principales son los árboles frutales cuya producción está destinada al mercado y, en menos cantidad, al consumo doméstico. Secundariamente consisten en hortalizas. Estos sistemas informan sobre ciertas costumbres. En Zacatecas, los huertos subsistieron hasta entrado el siglo xx y todavía se observan algunos y están estrechamente relacionados con las costumbres alimenticias, sobre todo en referencia a la preferencia por productos de las antiguas huertas. Alba González Jácome, "Breve historia de los huertos en México”, ponencia presentada en el Coloquio "México y sus historias: miradas múltiples" (México, Universidad Iberoamericana, 29 de agosto de 2012).

${ }^{19}$ D. Alonso de la Mota y Escobar, Descripción geográfica de los reinos de Nueva Galicia, Nueva Vizcaya y Nuevo León por D. Alonso de la Mota y Escobar, introd. de Joaquín Ramírez Cabañas, 2a. ed. (México: Pedro Robredo, 1940). 
complementarios. Lo anterior dio lugar a los primeros mayorazgos en la región, algunos de los cuales darían lugar a extensas haciendas (véase la figura 1 ).

Regulaciones iniciales del comercio

En 1575 los miembros de la diputación de minas señalaron la necesidad de realizar un tianguis o mercado en una zona delimitada donde se pudieran adquirir bastimentos básicos como granos. La intención era la regulación, y quien comercializara fuera del área sería acreedor a una multa de 20 pesos de minas aplicados por tercias (denunciador, prometido, susodicho y república). Señalaron el perímetro de plaza pública de minas en casas de Alonso de Herrera y Alonso Gutiérrez hasta las de los herederos de Diego Hernández de Proaño y la iglesia mayor. Para el real de Pánuco, el lugar designado era en la zona inmediata al rastro. ${ }^{20}$

El cabildo también mandó amojonar la legua circundante a la ciudad para evitar que hubiera ganado suelto pastando. Lo anterior daba seguimiento a las ordenanzas dictadas por Pedro Altamirano en 1585, las cuales tuvieron una aplicación ambigua, pues no se completó el proceso de autorización de la Audiencia. En las mismas ordenanzas, el visitador señaló la necesidad del establecimiento de una alhóndiga para que se pudiera hacer almacenamiento de bastimentos y limitar las alzas de precios propiciadas por acaparadores. ${ }^{21}$

Debido a las múltiples denuncias de mercaderes sobre los alguaciles de la ciudad de Zacatecas, el oidor y visitador general, Nuño Núñez de Villavicencio, señaló hacer públicas las ordenanzas sobre el comercio, pues los alguaciles argumentaban, en múltiples ocasiones, violación de sus directrices, pero sin mencionar los contenidos. Por tanto, mandó que se pregonaran cada año y se mostrarán a los solicitantes. ${ }^{22}$ Cabe señalar que las disposiciones mencionan sanciones económicas importantes para los alguaciles en caso de que abusaran de su autoridad.

${ }^{20}$ Archivo Histórico del Estado de Zacatecas (en adelante, AHEz), Ayuntamiento, Actas de Cabildo, primer libro, 1 de marzo de 1575 , f. $47 \mathrm{v}$.

${ }^{21}$ José Enciso Contreras, Ordenanzas de Zacatecas del siglo XVI y otros documentos normativos neogallegos, Serie Elías Amador (México: Ediciones del Ayuntamiento de Zacatecas; Zacatecas: Universidad Autónoma de Zacatecas, Facultad de Derecho, 1998), 8-19.

${ }^{22}$ AHEZ, Actas de Cabildo, Libro segundo, Ordenanzas, n. 1, 27 de julio de 1595, f. 114. 


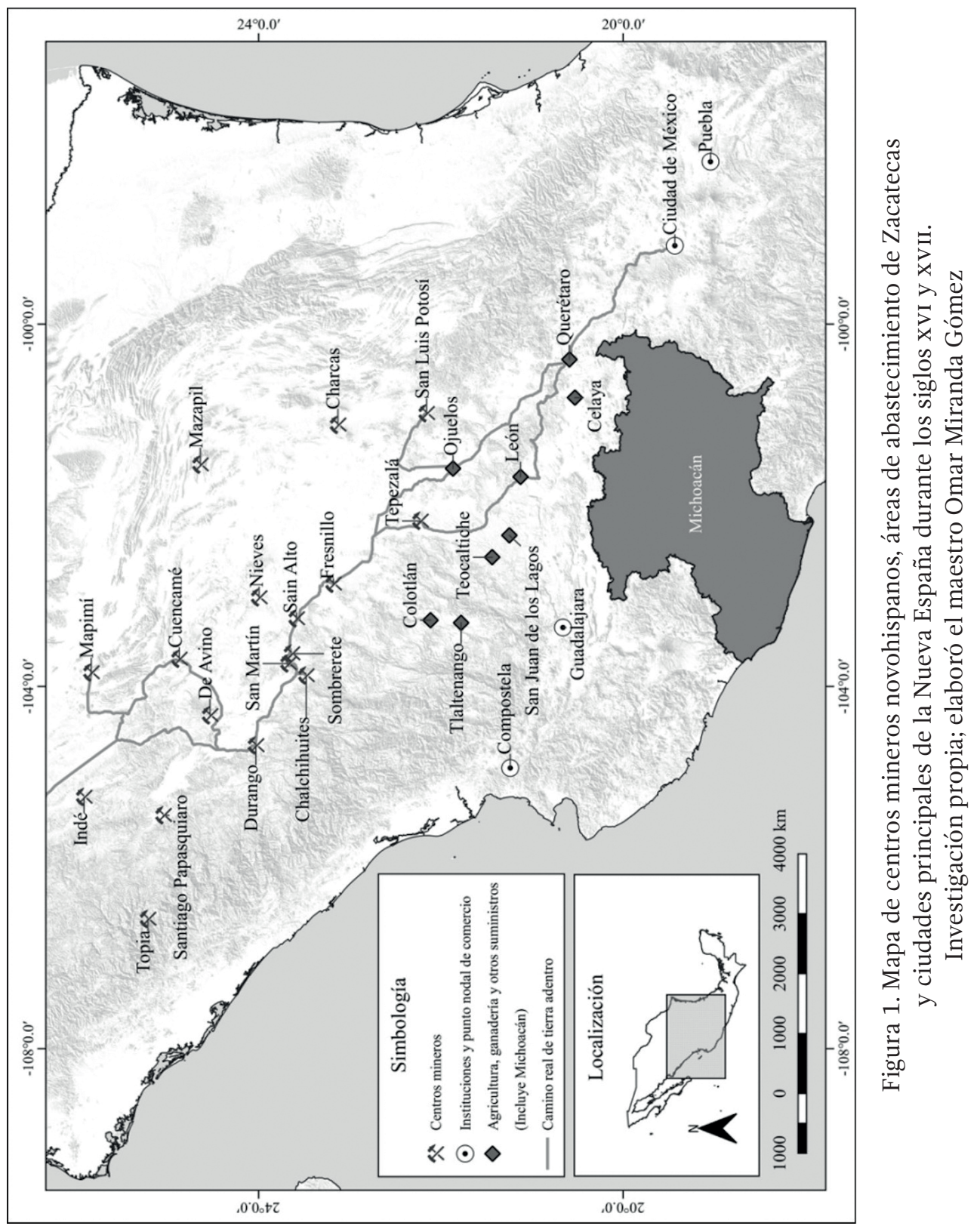


Como punto importante señalaba que los aranceles debían cobrarse entre dos o tres personas para mayor control, y cada uno debía llevar su registro. ${ }^{23}$ De igual forma se restringió que los alguaciles revisaran los libros de control de venta de productos de Castilla o productos de la ciudad de México, como jabón o cera. Por lo tanto, solamente se podía revisar lo relativo a comestibles y bebidas que se fueran a revender en la ciudad para tomar los derechos correspondientes. ${ }^{24}$

En abril de 1596, instruyeron que las posturas de bastimentos para la ciudad deberían presentarse los meses de mayo y junio para los productos del siguiente año. ${ }^{25}$

En lo que respecta a la regulación de los granos, en 1575 se discutió una importante alza en los precios del maíz. Si bien no se registra el precio alcanzado, se señalaba que los precios antes del alza oscilaban entre los $10 \mathrm{y}$ 12 reales por fanega. ${ }^{26}$ En septiembre de ese año, el cabildo mandó pregonar que los panaderos dieran tres panes de 12 onzas por un tomín y cada fanega de maíz a dos pesos de oro común. ${ }^{27}$

En octubre de 1576, en un acuerdo sobre el maíz, se señaló que el precio promedio era 9 a 11 reales por fanega; sin embargo, se había disparado por carestía. El cabildo instruyó buscar acaparadores y maíz que estuviera almacenado para ofrecerlo a la venta en precios máximos de dos pesos por fanega de maíz menudo y 14 tomines el maíz gordo de contado. ${ }^{28}$ En enero de 1584 continuó la carestía y la venta a precios excesivos. En esa ocasión se señaló que en los poblados circundantes se daban muchas reventas al estar menos vigilados. ${ }^{29}$

En 1593 el alza de precios y la leña cara afectaron a los panaderos (el quintal de harina a cuatro pesos de oro común). Por ello, se quejaron de no poder cumplir con las instrucciones de vender dos libras de pan cocido por un tomín. Por lo tanto, se autorizó el cambio a 28 onzas de pan por un tomín. ${ }^{30}$

En 1594, el cabildo instruyó a Juan de Monroy, mayordomo de la ciudad, que en su casa se preparara un quintal de harina para saber las libras de pan

\footnotetext{
${ }^{23}$ AHEz, Actas de Cabildo, Libro segundo, Ordenanzas, n. 4, 27 de julio de 1595, f. 116.

${ }^{24}$ AHEz, Actas de Cabildo, Libro segundo, Ordenanzas, n. 5, 27 de julio de 1595, f. 116.

25 AHEz, Actas de Cabildo, libro segundo, 9 de abril de 1596, f. 119.

${ }^{26}$ AHEZ, Actas de Cabildo, libro primero, 12 de marzo de1575, f. 48.

${ }^{27}$ AHEZ, Actas de Cabildo, libro primero, 13 de septiembre de 1575 , f. $54 \mathrm{v}$.

${ }^{28}$ AHEz, Actas de Cabildo, libro primero, 19 de octubre de 1576, f. $65 \mathrm{v}$.

29 AHEZ, Actas de Cabildo, libro primero, 23 de enero de 1584, f. 93v.

${ }^{30}$ AHEz, Actas de Cabildo, libro segundo, 7 de abril de 1593, f. 97.
} 
amasado y cocido que podían sacarse de ello y saber con mayor exactitud la conveniencia de las posturas. ${ }^{31}$

En 1600 el cabildo señalaba que, durante los primeros años del real de minas, los agricultores acudían directamente de los valles de Tlaltenango, Juchipila, Teocaltiche y Súchil para comerciar sus granos, pero en medio siglo las redes de intermediarios habían elevado los costos. ${ }^{32}$ A partir de 1620, se intensificó la discusión por el establecimiento de la alhóndiga para la ciudad. El corregidor instruyó que, provisionalmente, un jacal en la plaza principal hiciera las veces de este establecimiento, en lo que las finanzas de la ciudad permitían realizar la edificación. Para lo anterior, se solicitó que se entregaran 200 pesos en reales para el inicio de la construcción del edificio. ${ }^{33}$ En ese mismo año, se planteó el estanco de vino y azúcar, a fin de poder costear las obras públicas necesarias para la ciudad, como casas de cabildo, cárcel, alhóndiga, carnicerías y el arreglo de puentes. Para lo anterior, se solicitaba al gobernador y presidente de Audiencia el permiso correspondiente. ${ }^{34}$

A principios de enero de 1622, se registró una importante carestía de maíz, por lo que se señaló que los que llevaban a venta este fundamental producto deberían manifestar sus mercaderías ante el corregidor antes de su venta. ${ }^{35}$ En 1623, respecto al mantenimiento de la alhóndiga, hacen referencia a la fundación de la alhóndiga de San Luis y la retención de dos reales por carga de harina que se realizó en ese real para la edificación. ${ }^{36}$ La Audiencia de Guadalajara instruyó algo similar para la construcción de la alhóndiga de Zacatecas el 3 de noviembre de 1623, ${ }^{37}$ y para 1635 la alhóndiga ya funcionaba plenamente en la ciudad. ${ }^{38}$

Las primeras regulaciones para el establecimiento de alhóndigas se habían dado en la ciudad de México en $1584 .{ }^{39}$ La urgencia por el establecimiento de alhóndiga y pósito en la capital novohispana se debió a un

${ }^{31}$ AHEZ, Actas de Cabildo, libro segundo, 31 de agosto de 1594, f. 106.

${ }^{32}$ AHEZ, Actas de Cabildo, libro segundo, 7 de septiembre de 1600, f. 162-163.

${ }^{33}$ AHEZ, Actas de Cabildo, libro tercero, 6 de mayo de 1620, f. 54-54v.

${ }^{34}$ AHEZ, Actas de Cabildo, libro tercero, 27 de junio de 1620, f. 58v.

${ }^{35}$ AHEZ, Actas de Cabildo, libro tercero, 15 de junio de 1622, f. 100-101.

${ }^{36}$ AHEZ, Actas de Cabildo, libro tercero, 6 de septiembre de 1623, f. 118.

37 AHEZ, Actas de Cabildo, libro tercero, 6 de septiembre de 1623, f. 118v.

${ }^{38}$ AHEz, Actas de Cabildo, libro tercero, 27 de enero de 1635, f. 312v.

${ }^{39}$ Irene Vázquez de Warman, "El pósito y la alhóndiga en la Nueva España”, Historia Mexicana, v. 17, n. 3 (1968): 395-426. 
periodo de carestía y epidemias. ${ }^{40} \mathrm{La}$ alhóndiga sería una de las instituciones más características del mundo hispánico en cuanto a la administración y abasto de las ciudades.

Las iniciativas en San Luis Potosí datan de 1603, aunque el establecimiento efectivo se dio hasta $1609 .{ }^{41}$ A pesar de ser una fundación posterior a Zacatecas, San Luis estableció primero la alhóndiga y como se ha señalado se tomó su ejemplo para el financiamiento de la misma. La red de intermediarios que aumentaba los precios de los granos y que constantemente fue denunciada por el cabildo en Zacatecas seguiría operando hasta 1620. Entre 1614 y 1638 las regiones abastecedoras enfrentaron sequías y epidemias que quedaron registradas en las actas de cabildo. En enero de 1615 se dieron procesiones y novenarios en honor al Santo Cristo por pestes y sequía. En septiembre de ese mismo año, se acordó realizar una procesión en honor a san Nicolás Tolentino. Las sequías y epidemias sin duda aceleraron la instauración de la alhóndiga en la ciudad. ${ }^{42}$

Regulación del precio de vino y otros productos españoles en época de carestía

En agosto de 1575 la diputación acordó, debido a la sobreoferta de vino que existía en ese momento, que era necesario bajar los precios respecto de lo establecido en la postura previa, que había sido de 12 pesos la arroba y 3 tomines el cuartillo, a un máximo de 10 pesos la arroba y 2 1/2 tomines el cuartillo. ${ }^{43}$ El 19 de agosto de 1575 volvieron a bajar los precios de pan y vino. Las pipas de vino se vendían a 6 pesos la arroba al contado y fiado en 7 pesos y 2 tomines (y un máximo de 7 pesos y 3 tomines en esa modalidad). La harina en $2 \frac{1}{1} 2$ pesos el quintal. ${ }^{44}$ Sobre el pan y el vino, nuevamente se señaló que ambos productos se vendían a precios excesivos, pues estaba fijado que se vendiera a 6 pesos la arroba y en realidad se vendía a 8 pesos.

${ }^{40}$ José Armando Hernández Soubervielle, De piedra y maíz. Las alhóndigas y el abastecimiento de granos en San Luis Potosí durante el virreinato (México: El Colegio de San Luis, 2013), 29.

${ }^{41}$ José Armando Hernández Soubervielle, De piedra, 59.

42 AHEz, Actas de Cabildo, libro tercero, 3 de enero de 1614, f. 1v; AHEz, Ayuntamiento, Actas de Cabildo, libro tercero, 8 de septiembre de 1614, f. 11.

${ }^{43}$ AHEz, Actas de Cabildo, libro primero, 1 de agosto de 1575, f. 53.

${ }^{44}$ AHEZ, Actas de Cabildo, libro primero, 19 de agosto de 1575, f. 53. 
Se acordó el precio máximo de 8 pesos por arroba y el cuartillo a 2 tomines. De lo contrario se cobrarían 20 pesos de mina de multa aplicados en tercias. ${ }^{45}$

Se dio un periodo de suma escasez de vino a partir de junio de 1588 debido a la ausencia de la flota ese año. El cabildo tuvo que implementar diversas medidas, como rastrear las existencias y fijar precios máximos, ya que no se esperaba que hubiera mayores existencias hasta dentro de otro medio año. ${ }^{46}$ A Francisco Rosales se le encontraron aproximadamente 30 pipas y se le fijó un precio máximo de 16 pesos de oro común por cada arroba junta o por menudeo, para todo el vino que tuviera; además, el de él debía venderse con prioridad para reservar el que se fuera encontrando conforme pasaran los meses previos a la regularización. ${ }^{47}$ El 8 de noviembre de 1588 la carestía de vino se incrementó, de tal forma que Alonso Canto presentó una postura que le permitió introducir 16 pipas de vino procedentes de la ciudad de México. Éstas podrían venderse en un precio máximo de 16 pesos la arroba. Se le prohibió vender a indios, mulatos o negros, y por tanto la mercancía quedaría reservada a españoles solamente. ${ }^{48}$

El 12 de agosto de 1588 se entregó al receptor de alcabalas la instrucción de implementar la exención mandada por el virrey Martín Enríquez de Villamanrique, donde se señalaba que no debía cobrarse alcabala sobre la harina vendida en tianguis y plazas públicas. ${ }^{49}$

En marzo de 1590, los mercaderes de la ciudad solicitaron al cabildo poder aumentar el precio del vino. Argumentaban pérdidas económicas debido a que el precio máximo fijado en ese momento era 10 pesos, que era el monto en que los comerciantes lo adquirían debido a la ausencia de la flota y a que los productos castellanos como el vino habían alcanzado un alto precio en la ciudad de México. Respecto de esto, el factor Alonso Caballero señaló que le constaba que el vino en la ciudad de México valía a 2.5 reales, y por tanto, "el que lo trae a esta ciudad ha de ganar algo". ${ }^{50}$

En julio de 1593 continuó la carestía de vino, y ante eso, el cabildo mandó que se almacenaran 20 pipas para resguardo. El precio máximo,

${ }^{45}$ AHEz, Actas de Cabildo, libro primero, 19 de agosto de 1575, f. 53.

${ }^{46}$ AHEz, Actas de Cabildo, libro segundo, 1 de junio de 1588, f. 31v.

${ }^{47}$ AHEZ, Ayuntamiento, libro segundo, 1 de junio de 1588, f. $31 \mathrm{v}$.

${ }^{48}$ AHEz, Actas de Cabildo, libro segundo, 8 de noviembre de 1588, f. 36v.

${ }^{49}$ AHEz, Actas de Cabildo, libro segundo, 11 de julio de 1588, f. 41-42. Se señala que la instrucción había sido entregada el 13 de marzo de 1575 y que antes de eso no había sido implementada, f. 34v-35.

${ }^{50}$ AHEz, Actas de Cabildo, libro segundo, 9 de febrero de 1590, f. 78-79. 
fijado en ese momento por el cabildo, era de 4 tomines por cuartillo. En alguna inspección se notó que el vino almacenado comenzaba a avinagrarse, y para no perder la mercancía, se tomó la decisión de venderlo en remate público. ${ }^{51} \mathrm{Al}$ comerciante Hernán Gómez de Giles se le hallaron en su bodega cuatro pipas, las cuales, como instruyó el cabildo, debía vender en la ciudad y a un precio acordado, so pena de multa de 100 pesos por venta previa, o de 500 si lo sacaba de la ciudad. ${ }^{52}$

Nuevamente, hacia 1596, no llegarían productos de Castilla como vinos, aceite, azafrán, hierro y papel, entre otras mercaderías. ${ }^{53}$ El corregidor realizó búsquedas, y por lo tanto, se señaló que había en existencia vino y aceite. El flujo de aceite, vino, azafrán, ruanes, papel, hierro y otras mercancías hacia el interior de la ciudad no se había suspendido, aunque los regatones habían causado gran daño con la especulación y la subida de precios en los periodos anteriores de carestía.

Instruyeron, respecto de los vinos, las siguientes compras a comerciantes, de acuerdo con los precios adquiridos por ellos: 14 pipas de vino de Pedro Torres de la Cueva; ocho pipas a Juan Marmolejo Tinojo; cuatro a Francisco Pérez de Pedro de San Martín y a Andrés de Quesada; y dos pipas a Francisco de Fuentes de Bartolomé de Aspitia. Esta mercancía debía estar almacenada para venta a los vecinos de la ciudad a precios moderados y convenientes. Fijaron el precio máximo en tabernas en tres tomines el cuartillo y se refrendaba la restricción de venta a negros, indios y mulatos. ${ }^{54}$

Asimismo se prohibió la salida de vino de la ciudad y debían declararse ante el teniente las ventas realizadas. De igual forma, se prohibió la salida de aceite, hierro, papel, azafrán, ruan, almendras, pasas, aceitunas, herraje y otros efectos de comer y mercancía proveniente de Castilla (véase el cuadro 2). ${ }^{55}$ En octubre de 1596 se solicitó al teniente y los regidores que reforzaran la cala, la cata y las restricciones de salida de mercancía de la ciudad. ${ }^{56}$ En noviembre de 1596 se pidió a los mercaderes vender con moderación sus mercancías, debido a la carestía y los sobreprecios que se podían originar si vendían todas sus existencias. ${ }^{57}$

${ }^{51}$ AHEZ, Actas de Cabildo, libro segundo, 7 de abril de 1593, f. 98-99.

52 AHEz, Actas de Cabildo, libro segundo, 7 de abril de 1593, f. 98-98.

${ }^{53}$ AHEz, Actas de Cabildo, libro segundo, 8 de octubre de 1596, f. 122v.

${ }^{54}$ AHEZ, Actas de Cabildo, libro segundo, 8 de octubre de 1596, f. 123.

55 AHEz, Actas de Cabildo, libro segundo, 8 de octubre de 1596, f. 123.

56 AHEz, Actas de Cabildo, libro segundo, 30 de octubre de 1596, f. 127.

${ }^{57}$ AHEz, Actas de Cabildo, libro segundo, 9 de noviembre de 1596, f. 129. 
Cuadro 2

PRECiOS DE MERCANCÍAS EUROPEAS, NOVIEMbRE DE 1596

\begin{tabular}{llc}
\hline \multicolumn{1}{c}{ Producto } & Cantidad & Precio \\
\hline Vino & Cuartillo & 3 reales $1 / 2$ \\
& Cuartillo & 3 reales $1 / 2$ \\
Aceite & Media arroba & $51 / 2$ pesos \\
Hierro & Quintal & 4 pesos \\
Azafrán & Onza & 10 tomines \\
Ruan de fardo & Vara & 13 tomines \\
Papel & Mano & 4 tomines \\
Almendra & Libra & 1 1/2 peso \\
Pasas de Almuñécar & Libra & 6 tomines \\
\hline
\end{tabular}

Fuente: AHEZ, Ayuntamiento, Actas de Cabildo, 15 de noviembre de 1596, f. 130.

Los precios autorizados de productos europeos aprobados por el cabildo en 1596 fueron los siguientes:

En julio de 1597 la carestía de vino continuaba y el precio del cuartillo de vino se mantuvo en 3 reales y medio. El corregidor Nicolás Rodríguez efectuó una visita a las tabernas y señaló que en muchas había existencias, pero no se vendía hasta que los habitantes estuvieran dispuestos a pagar precios mayores a los fijados por el cabildo. ${ }^{58}$ La instrucción fijada por el cabildo fue la inspección semanal de las existencias de vino embodegadas para tener mejor control de las salidas. Sin embargo, eso no fijaba controles para los taberneros y también se puede aseverar que había mercancía que no había sido detectada por el cabildo que entraba a la ciudad y que permitía que los mercaderes tuvieran existencias suficientes. Lo anterior facilitó la subida de precios no autorizada por el cabildo. Ante tal número de irregularidades, y con recursos limitados para operación, los miembros del cabildo optaron simplemente por imponer vigilancia en algunas áreas que podían controlar, como las existencias de lo confiscado, pero no podían tener un absoluto control del comercio en una ciudad con auge económico en una época de carestía.

En abril de 1603, el cabildo acordó solicitar a Francisco Quintana Dueñas, juez que se encontraba en la ciudad, que parte de la sisa del vino sirviera para los propios de la ciudad por un periodo de 10 años, ante la carencia de

58 AHEz, Actas de Cabildo, libro segundo, 27 de junio de 1597, f. 135. 
fondos propios. Se estimaba que se obtendrían unos 4000 pesos por año de esa forma. ${ }^{59}$ En noviembre de 1608, se comunicó una cédula que autorizaba a la ciudad la mitad de lo recaudado por la sisa. Se explicaba que, como antecedente durante el siglo XVI, esa cantidad se concedió a la ciudad para gastos de seguridad. ${ }^{60}$

En acuerdo de cabildo del 22 de agosto de 1615 fue que se pregonaría públicamente que los mercaderes que comercializaran vino debían manifestar la cantidad de arrobas de vino vendidas a rescatones para venta al menudeo, para el pago de la sisa correspondiente. ${ }^{61}$

En un auto referente a la visita realizada por el oidor Diego Medrano en 1620 , se señalaba que a los taberneros constantemente se les imponían multas en las revisiones del cabildo. Aunque éstas eran excesivas, reconocía que la venta de vino a los indios y la incursión de vino de cocos eran comunes en la ciudad. ${ }^{62}$

Suponemos, debido a la importancia que los miembros del cabildo le conceden a este producto, que existió un amplio mercado de vino de contrabando. Debido a las características de la ciudad y la minería como actividad articuladora, el consumo de vino no lo clasificamos como producto de lujo, sino como un producto de extendido consumo. Hay estudios que nos muestran la tolerancia en el consumo de alcohol, ${ }^{63}$ lo anterior unido a los beneficios por la recaudación fiscal fueron factores que incentivaron su comercialización, aunque se planteaba un consumo diferenciado con una prohibición de consumo de vino por parte de los indígenas. Tal reglamentación fue continuamente traspasada en Zacatecas, donde consta en múltiples testimonios del cabildo que los establecimientos no respetaban esta prohibición. Ante la carencia de pulques en la región, al incentivo en la recaudación fiscal y al mercado que podía tener los productos alcohólicos, no es extraño encontrarnos con un activo comercio de vino de cocos del Pacífico. ${ }^{64}$

59 AHEZ, Actas de Cabildo, 1603, f. 172-172v.

${ }^{60}$ AHEZ, Actas de Cabildo, 10 de noviembre de 1608, f. 221-221v.

${ }^{61}$ AHEZ, Actas de Cabildo, 22 de agosto de 1615, f. 11v.

${ }^{62}$ AHEz, Actas de Cabildo, 1620, 15 de julio de 1620, f. 60v.

${ }^{63}$ Paulina Machuca Chávez, "Alcohol, mineros y operarios en la Nueva España (siglos XVI-XVIII)", en Alcohol y trabajo en América Latina. Siglos XVII-XX, coord. de Patricio Herrera González y Juan Carlos Yáñez Andrade (Valparaíso: América en Movimiento Ediciones; Valparaíso: Universidad de Valparaíso, Centro de Investigación en Innovación, Desarrollo Económico y Políticas Sociales, 2019), 17-41.

${ }^{64}$ El reciente estudio de Paulina Machuca arroja importantes avances en cuanto a la producción y distribución de este producto. Paulina Machuca, El vino de cocos en la Nueva 


\section{Organización del abasto de carne}

La figura del obligado de carnes fue un esquema importado de la península ibérica, en el que un señor de ganados se comprometía a abastecer las carnicerías de determinada ciudad a un precio fijo por un periodo mínimo de un año. ${ }^{65}$ Los abastos de carne de los importantes centros urbanos eran responsabilidad de sus respectivos cabildos. Debido a la rápida multiplicación del ganado, durante los primeros tiempos, la figura del obligado de la carne pudo no haber existido en la Nueva España. Sin embargo, los cabildos implementaron los contratos para asegurar las cuotas e impuestos necesarios para que funcionase adecuadamente la administración de las ciudades, así como para mantener un abastecimiento continuo de carne a la población, a precios fijos y accesibles.

En el caso de Zacatecas, la minería fue fundamental, pero tanto el desarrollo de ésta como la supervivencia de la población, estuvieron ligados a la ganadería y a la agricultura. En un primer momento, la posesión de tierras se vio como el medio necesario para la producción de bastimentos para las minas:

La agricultura y la ganadería, que llegaron a ser más tarde los principales recursos de los poderosos, no constituyeron al principio más que una necesidad para el aprovisionamiento de las minas, y luego oportunidades de inversión para el dinero

España. Historia de una transculturación en el siglo XVII (Zamora, Michoacán: El Colegio de Michoacán, 2018).

${ }^{65}$ En el siglo xv ya existía la figura del obligado en la península ibérica, pero generalmente era ocupada por los carniceros de la villa o ciudad, aunque el cargo comenzaba a ser desempeñado en mancomunidad por sujetos que no se dedicaban directamente a la ganadería. Los fiadores proporcionaban el ganado cuando los obligados se veían rebasados por la demanda de la población. Los miembros del Ayuntamiento constantemente supervisaban a los obligados y también vigilaban que no faltaran provisiones a la población. En años de escasez el Ayuntamiento podía concederles un permiso para venta a mayor precio (siempre que se hubiera evaluado la situación). En 1490 en Madrid se acordó hacer un matadero único para la villa, a fin de regular mejor la calidad de las carnes. En el siglo xvi, con la llegada de los Austrias, el abasto comenzó a ser rematado entre los notables de la villa o ciudad. Estos obligados pudieron llegar inclusive a obtener préstamos para el abasto a un interés del 10\% anual. En 1573 se acordó que el Ayuntamiento llevara un registro de los gastos entre los que se encontraban los propios de la villa o ciudad. La temporalidad del relevo variaba de poblado a poblado, ya que en Madrid iba del día de San Juan (24 de junio) al año siguiente. Antonio Matilla Tascón, Abastecimiento de carne a Madrid, 1477-1678 (Madrid: Instituto de Estudios Madrileños, 1994), 10-62. 
ganado en otras formas: la operación era tentadora, a causa de los precios altísimos que alcanzaban los víveres y las bestias de carga en los nuevos centros mineros. ${ }^{66}$

Se tiene conocimiento de la amplia circulación de productos ganaderos durante el siglo XVI en los reales de minas de Zacatecas, y de la alta concentración de tierras por parte de algunos de los primeros colonos; sin embargo, no hay datos suficientes para hacer un rastreo de los precios y ganaderos que lograron los abastos. No obstante, hay algunos referentes.

Hacia los años de 1572 y 1573 se menciona por primera vez la figura del obligado. Aunque no se dan a conocer sus nombres, se señala que en los contratos quedó especificado que éste se encargaría de elaborar un edificio de tapias, tajamanil y otras reparaciones para el rastro de las minas. Sin embargo, en un acto de posesión realizado posteriormente, se evidenció que no se llevaron a cabo dichas reparaciones, por lo que se exigió tanto el pago correspondiente por reparaciones como el abono de los 500 pesos del prometido para el periodo. ${ }^{67}$ A pesar de que se desconoce el nombre del obligado para esos años, se señaló que el de los años 1572-1573 no era criador ni tenía estancias, por lo que se le permitió tener el ganado en dos estancias pertenecientes a las minas, las denominadas de Los Machines y Cieneguilla de los Carneros (cercanas a la zona de huertas). Como ya estaban despobladas, y las minas tenían necesidad de recursos, se señaló la necesidad de venderlas. Para ello se pregonaría y se recibirían posturas. ${ }^{68}$

En 1575 se nombró a Domingo de Gorraz obligado de las carnicerías. La libranza la dio Alonso de Herrera por 350 pesos. ${ }^{69}$ Para el mismo año se nombró a Hernando de Paz como veedor de carnicerías. ${ }^{70}$ La carencia de fondos por parte de la ciudad condicionó que no siempre se nombraran veedores y la vigilancia se realizó de forma parcial por parte del ayuntamiento. ${ }^{71}$

La mesta era una organización de la península ibérica, mediante la cual los ganaderos llevaban a pastar sus ganados a regiones alejadas en época de carestía. Es importante señalar que, aunque en la Nueva España se estableció formalmente esta institución, en el septentrión no tuvo mucho arraigo,

${ }^{66}$ François Chevalier, La formación de los latifundios en México. Haciendas y sociedad en los siglos XVI, XVII y XVIII (México: Fondo de Cultura Económica, 1999), 210.

${ }^{67}$ AHEz, Actas de Cabildo, primer libro, 8 de abril de 1575, f. 50.

${ }^{68}$ AHEz, Actas de Cabildo, primer libro, 8 de abril de 1575, f. 50v.

${ }^{69}$ AHEz, Actas de Cabildo, primer libro, 8 de abril de 1575, f. 50v.

${ }^{70}$ AHEz, Actas de Cabildo, primer libro, 8 de abril de 1575, f. 50v.

${ }^{71}$ AHEz, Actas de Cabildo, libro segundo, 23 de enero de 1587, f. 8. 
debido a la baja densidad demográfica de estas zonas y a la alta concentración de tierras por parte de los ganaderos del norte. ${ }^{72}$ Lo anterior se confirma con la respuesta a la instrucción girada desde la ciudad de México, para el establecimiento de alcaldes de mesta, donde se señala que tal institución no existía en la ciudad. ${ }^{73}$

Pedro de Umendia fue el obligado del abasto de carnes en el año de $1590 .{ }^{74}$ La ciudad continuó dependiendo de forma importante de los prometidos de los obligados para el pago de salarios y diligencias a la ciudad de Guadalajara, para enviar emisarios por asuntos con la Real Audiencia, o para reparaciones de la ciudad, tal como la de los puentes de la calle de Tacuba, pagados por el obligado de 1592, Pedro Venegas. ${ }^{75}$

Para establecer algunos puntos de comparación, se puede hacer referencia a los trabajos de Ward Barrett ${ }^{76}$ y José Matesanz. ${ }^{77}$ El primero señala que en los contratos de abastecimiento de cárnicos a Cuernavaca se anexaron lugares circunvecinos. De forma similar, el abasto de Zacatecas incluyó Pánuco y Vetagrande. Al igual que en Cuernavaca había comercio ilegal de carne y sacrificios en lugares no autorizados y fue una circunstancia que atravesaron los cabildos novohispanos durante el periodo. En Zacatecas, los mineros podían tener ganado propio para sacrificio debido a la alta demanda de sus productos en estas zonas.

Ward Barret señala aprovisionamientos de carne recibidos por los operarios y esclavos. Aunque no contamos con datos para comparar esta situación, los reales de minas novohispanos y especialmente los que se localizaron en Zacatecas y Durango tuvieron una alta población flotante para la producción, y con sus salarios y partidos podían aprovisionarse de cárnicos y otros alimentos debido a la gran demanda calórica que demandaba su trabajo en minas. Mota y Escobar indica que el salario de los indios barreteros

${ }^{72}$ Para ver más detalles sobre la mesta en la Nueva España, véase Willliam Dusenberry, The Mexican Mesta. The Administration of Ranching in Colonial Mexico (Urbana: University of Illinois Press, 1963).

${ }^{73}$ AHEz, Actas de Cabildo, 1 de enero de 1588, f. 22-22v.

${ }^{74}$ AHEZ, Actas de Cabildo, 13 de agosto de 1590, f. 70.

75 AHEz, Actas de Cabildo, 14 de abril de 1592, f. 87v.

${ }^{76}$ Ward Barrett, "The Meat Supply of Colonial Cuernavaca", Annals of the Association of American Geographers, v. 64, n. 4 (diciembre 1974): 525-540, doi: 10.1080/00045 605209352144 .

77 José Matesanz, “Introducción a la ganadería en Nueva España, 1521-1535”, Historia Mexicana, v. 14, n. 4 (1965): 559. 
en Zacatecas a inicios del siglo XVII iba de 5 a 8 pesos, más lo que pudieran acumular con el sistema de partido. ${ }^{78}$

En los contratos de abasto de carne del siglo XVII con que contamos consta que las vísceras eran un producto de alto consumo, situación que obedeció probablemente a los requerimientos calóricos antes mencionados. El precio de este producto se mantuvo constante en un real durante 1612 y 1648.

Otro de los insumos de particular importancia fue el sebo, que era utilizado para la elaboración de velas domésticas y para iluminación de las galerías al interior de las minas en el periodo señalado, y se mantuvo altamente fluctuante, entre 3 y 6 pesos por arroba. ${ }^{79}$ Las posturas, estuvieron poco estandarizadas y tuvieron variaciones importantes de un año a otro. En la misma situación se encontraban los cueros de res, que constituían un producto de particular importancia durante ese tiempo debido a su amplia utilización y cuyo precio varió de 1 peso a 15 reales durante el periodo 1612-1648.

En cuanto a los precios de la carne, en Zacatecas la de carnero tuvo más especificaciones en sus posturas que las de res, lo que puede suponer un amplio consumo y el precio de la carne de cordero fue incrementando respecto del de la de res. Desde siglo XviI, el ganado ovino fue conformando una especialidad ganadera en el norte de la Nueva España, lo cual tuvo como resultado una masiva exportación de esta producción a mercados urbanos de gran importancia como ciudad de México, Puebla y Guanajuato en el siglo XVIII. ${ }^{80}$

Barrett no señala claramente la relación unidad-precio, aunque lo más probable es que las posturas estuvieran vinculadas a libras por real para la res y onzas para el carnero. ${ }^{81} \mathrm{El}$ periodo en el que se puede establecer una comparación entre Zacatecas y Cuernavaca comprende 1643 a 1648 (véase el cuadro 3). Se aprecia que la cantidad de carne de res ofrecida en $\mathrm{Za}$ catecas era significativamente mayor por real que la que se ofreció en Cuernavaca, ya que fue de 10 libras por real contra 12 y hasta 15 libras por real en el mismo periodo en Zacatecas. Por el contrario, los precios del carnero

${ }^{78}$ Mota y Escobar, Descripción, 151.

${ }^{79}$ AHEz, Abasto de Carnes, caja 1, 1612-1648.

${ }^{80}$ Enriqueta Quiroz, Entre el lujo y la subsistencia: mercado, abastecimiento y precios de la carne en la ciudad de México, 1750-1812 (México: El Colegio de México; México: Instituto de Investigaciones Dr. José María Luis Mora, 2005).

${ }^{81}$ Barrett, "The Meat", 532. 


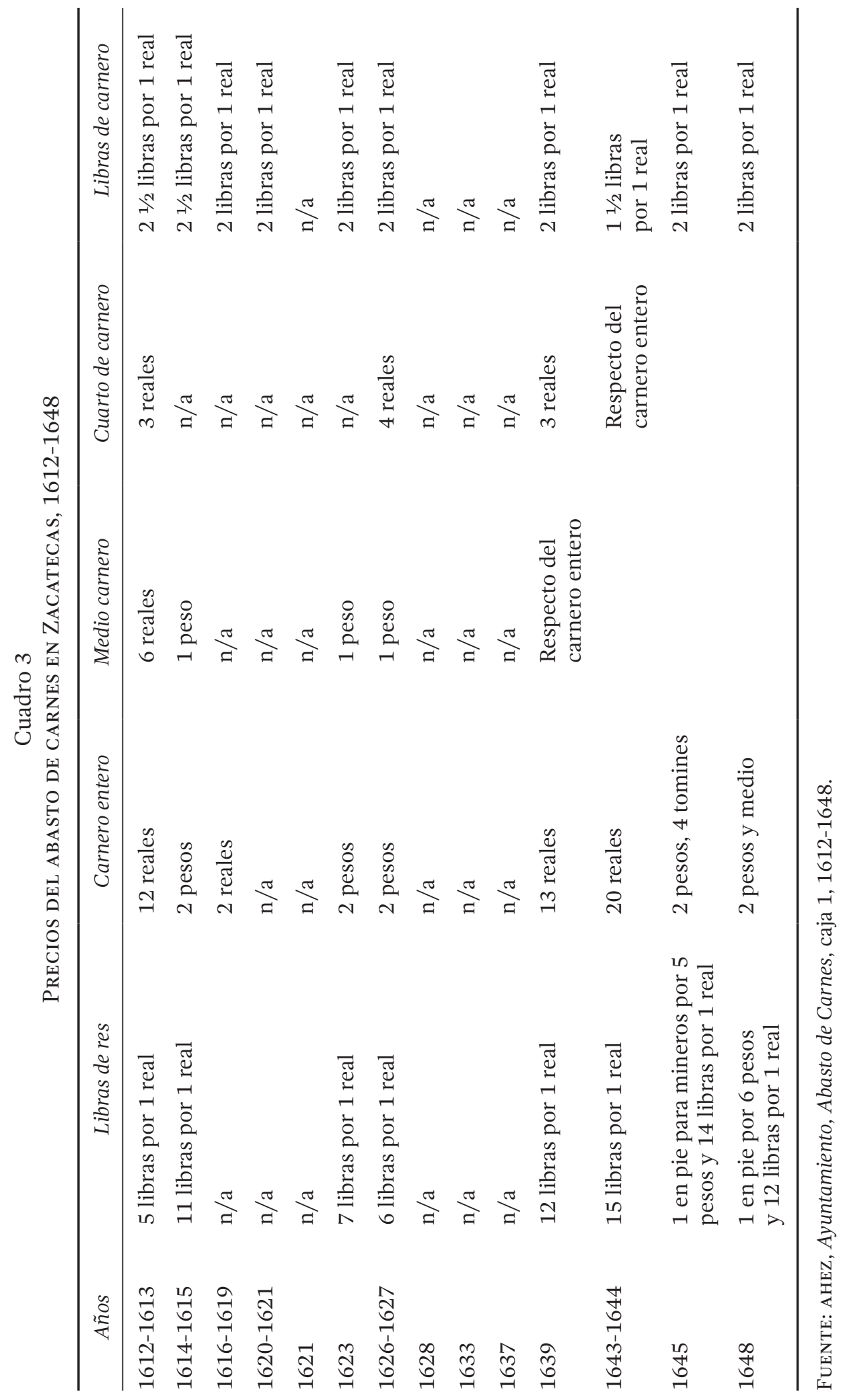


se encontraban en condiciones similares; en Zacatecas iban de 2 a 2.5 libras por real y en Cuernavaca de 40 a 32 onzas por real.

Los precios proporcionados por Matesanz son del siglo XVI y muestran que en los inicios de la ciudad de México las posturas de carne no diferenciaban entre res y carnero, y en esa etapa inicial también existieron posturas de cerdo e inclusive de carne de venado. Las negociaciones se establecieron a partir de arreldes que equivalen a 4 libras aproximadamente. Los precios entre 1524 y 1532 fluctuaron entre 6 reales por arrelde hasta 1 arrelde por un real, lo cual se explica por el auge inicial de la ganadería en las inmediaciones de la ciudad de México. ${ }^{82}$

\section{Recursos forestales}

Durante el recorrido del obispo Alonso de la Mota y Escobar, se dejó constancia de la rápida depredación forestal de los bosques en la zona de Zacatecas, por la irrupción de la minería y el poblamiento que devino de ella.

Había en su descubrimiento mucha arboleda y monte en estas quebradas, las cuales todas se han acabado y talado/ con las fundiciones, de manera que si no son unas palmillas silvestres, otra cosa no ha quedado. Y así es la leña muy cara en esta ciudad porque se trae de ocho a diez leguas en carretas. Era todo este ámbito de serranía y bosque en el tiempo de la gentilidad, el más famoso coto de corzos, liebres, conejos, perdices y palomas que tenía ningún señor en el mundo, y así gozaban de él los señores y caciques que lo poseían, cuya nación y vasallos se llamaban zacatecos, de cuyo nombre se le quedó a esta ciudad de las Zacatecas. ${ }^{83}$

La introducción del método de amalgamación se dio a partir de 1557; sin embargo, los insumos madereros siguieron usándose en la extracción y procesamiento, al tiempo que no todos los mineros cambiaron al método de amalgamación de forma inmediata, ${ }^{84}$ a pesar de que se facilitó el suministro de azogue y también se abarataron los costos, pues hasta las primeras décadas del siglo XVII, el mercurio se vendió a crédito. ${ }^{85}$

82 Matesanz, “Introducción”, 559.

${ }^{83}$ Mota y Escobar, Descripción, 140.

${ }^{84}$ Lacueva, "Zacatecas", 545.

${ }^{85}$ Lacueva, "Zacatecas", 552. 
Liberados los mineros de la carga que suponía buena parte de la financiación del mercurio, dos factores más contribuyeron decisivamente al auge de la producción de plata que viviría Zacatecas desde comienzos del siglo XVII. El primero fue el fin de la guerra chichimeca, y el segundo las mejoras técnicas que aportó la experiencia acumulada tras cuatro décadas de empleo de la técnica de amalgamación. ${ }^{86}$

A pesar de eliminar un poco la necesidad del consumo de leña mediante la introducción de la nueva tecnología de amalgamación, el proceso de patio de todas formas requería madera, lo que repercutía en la deforestación del entorno inmediato. De la Mota habla de los altos costos de la madera para inicios del siglo XVII. ${ }^{87}$ El estudio de Studnicki y Schecter estima que la deforestación durante el periodo virreinal en Zacatecas fue de $67854 \mathrm{~km}^{2}{ }^{88}$ El hecho de que la tala fuera tan elevada se debió a que hasta fines del siglo XIx la madera fue el único mecanismo de combustión, la cual era fundamental tanto para labores domésticas como para industrias de todo tipo.

Studnicki y Schecter se refieren a la lucha de dos agroecologías o modelos de poblamiento en el siglo XVI. Por una parte, el de los pueblos originarios del septentrión novohispano, quienes después de siglos de convivir en este territorio tenían un modelo basado en ocupación temporal y caza y recolección, y por otra, el modelo peninsular que se originó a partir de los descubrimientos argentíferos. Éste se basaba en la agricultura extensiva y el pastoreo. Lo anterior, aunado a la tala extensiva por presión demográfica y a la combustión para beneficio de metales, ocasionó la destrucción del sistema ecológico moldeado por las comunidades originales. ${ }^{89}$

${ }^{86}$ Lacueva, "Zacatecas”, 553.

${ }^{87}$ Mota y Escobar, Descripción, 556.

${ }^{88}$ Daviken Studnicki-Gizbert y David Schecter, "The Environmental Dynamics of a Colonial Fuel-Rush: Silver Mining and Deforestation of New Spain, 1522 to 1810”, Environmental History (Oxford, Oxford University Press on behalf of Forest History and American Society for Environmental History), v. 15, n. 1 (enero 2010), 94.

${ }^{89}$ Studnicki-Gizbert, "The Environmental”, 109. El estudio llega a las siguientes conclusiones: "Por último la creación de la agroecología colonial alrededor de los centros mineros novohispanos repercutió en la deforestación por el impulso de la minería. Los campos de sembradío y pastoreo, en las minas y las fundiciones intervinieron en el proceso natural de reforestación. Cuando la minería aumentó su capacidad de producción en el siglo XVIII esta dinámica se aceleró" ["Finally, the creation of a colonial agroecology around the mining centers of New Spain feedback into the dynamics of mining-driven deforestation. The extension of fields and pasturages around the mines and smelters blocked the processes of natural afforestation. Thus Mexican silver production accelerated in the eighteenth century, cut down in previous centuries”]. Studnicki-Gizbert, “The Environmental”, 210. 
Se aprecia, en los miembros del cabildo y en los oidores de la Audiencia de Nueva Galicia que pasaron por la zona, la preocupación que la rápida deforestación ocasionó, ya que en las ordenanzas dejaron previsiones para aminorar esta situación. ${ }^{90}$ Específicamente en las dictadas por Francisco de Mendiola, ${ }^{91}$ donde se prohibía la tala extensiva durante toda la semana. También exigía esfuerzos a las autoridades locales para eliminar los hornos portátiles denominados cendradillas, que eran sumamente populares entre los mineros para poder beneficiar mineral del partido. ${ }^{22}$ También se pedía limitar la tala indiscriminada por medio del corte de ramas y evitar el de troncos, además se proponía que el corte libre en montes para reventa fuera suprimido. Se hizo especial atención en la conservación de los encinos,

Otrosí, porque según soy informado se tiene por experiencia y consta de la información ante mí presentada, que los encinos y árboles de esta comarca cortándoseles las ramas y que cuando troncones altos no turnan a echar nuevas, antes se secan y pierden y los que se cortan bajos vuelven a renovar y echar ramas, prohíbo y defiendo que de aquí adelante ninguna persona corte los tales encinos y árboles por lo alto ni las ramas, sino junto a la raíz y haz de la tierra, porque así conviene al aumento y conservación de los montes y perpetuidad de esta población; so pena de cada veinte pesos de oro, aplicados como dicho es. ${ }^{93}$

Era obligación de las autoridades locales tener guardabosques. ${ }^{94} \mathrm{La}$ disminución de los bosques fue un fenómeno temprano descrito por el primer virrey Mendoza, quien señaló la situación y mencionó la existencia de ordenanzas al respecto. Andrés Lira señala que la monarquía española tenía reglamentaciones sobre la conservación de bosques; ${ }^{95}$ sin embargo,

${ }^{90}$ Enciso, Ordenanzas, 80.

${ }^{91}$ Traslado bien y fielmente sacado de unas ordenanzas que hizo en las minas de los Zacatecas, el señor licenciado Francisco de Mendiola, oidor alcalde mayor de la real audiencia de este reino de Galicia, según que por ellas parecía su tenor del cual es este que se sigue. Zacatecas, 6 de marzo de 1568, AGI, Guadalajara, R12, No. 21-23, en Enciso, Ordenanzas, 82-106.

92 Enciso, Ordenanzas, 92.

93 Enciso, Ordenanzas, 93.

${ }^{94}$ Enciso, Ordenanzas, 94.

95 No han sido localizadas las ordenanzas mencionadas por Mendoza, pero existe el antecedente de la pragmática de los Reyes Católicos de 1496, donde se menciona que solamente debían cortarse maderas de los bosques densos y preferentemente las ramas para favorecer el crecimiento y la conservación. Andrés Lira, "Los bosques en el virreinato 
en las regiones mineras se impusieron las necesidades inmediatas y en el siglo XVI el corte inmoderado para uso doméstico y la transformación de la plata tuvo un efecto en la densidad de los bosques circundantes.

La modificación del paisaje a partir del poblamiento de los reales de minas queda evidenciado, como se ha señalado, en descripciones de la época; sin embargo, es necesario estudiar con mayor atención el cambio de capa vegetal y las estrategias adaptativas que la sociedad de su tiempo adoptó ante los cambios ambientales que sucedieron.

\section{Consideraciones finales}

El rápido poblamiento de Zacatecas conllevó una importante tarea de organización, tanto para el inicio de las actividades extractivas y de transformación de la plata como para el abastecimiento de productos básicos al nuevo real de minas. Desde etapas tempranas se conformaron zonas productoras de granos, y el abasto de ganado para consumo humano y bastimentos de la minería quedó garantizado con la ganadería extensiva que se dio en el área inmediata, dando su inicio en el norte novohispano.

La diputación de minas y, posteriormente, el cabildo de la ciudad realizaron importantes esfuerzos en la regulación del comercio y en el control de precios de productos básicos en época de carestía. Sin embargo, el alejamiento de Zacatecas conllevó a que el control de precios ante la carestía fuera limitado.

Por último, los recursos forestales originarios se vieron afectados de forma temprana. La tala, el pastoreo y la agricultura modificaron el paisaje de la región. La legislación y algunas descripciones dejaron huella de las tensiones sociales que la deforestación tuvo en su momento, y de algunas medidas que se dictaron con el ánimo de paliar el rápido cambio de capa vegetal que se estaba dando.

(Apuntes sobre la visión política de un problema)", Relaciones. Estudios de Historia y Sociedad, v. 11, n. 41 (1990): 119. 


\section{FUENTES}

Fondos documentales

Archivo Histórico del Estado de Zacatecas (AHEz), Fondo Ayuntamiento.

\section{Bibliografía}

Arlegui, José de. Crónica de la Provincia de San Francisco de Zacatecas. México: José Bernardo de Hogal, 1737.

Acuña, René, ed. Relaciones geográficas del siglo XVI. Nueva Galicia. México: Universidad Nacional Autónoma de México, 1988.

Bakewell, Peter J. Minería y sociedad en el México colonial. Zacatecas 1546-1700. México: Fondo de Cultura Económica, 1971.

Barrett, Ward. "The Meat Supply of Colonial Cuernavaca." Annals of the Association of American Geographers, v. 64, n. 4 (diciembre 1974): 525-540, doi: $10.1080 / 00045605209352144$.

Calvo, Thomas. Por los caminos de Nueva Galicia. Transportes y transportistas en el siglo XVII. México: Universidad de Guadalajara; México, Centro Francés de Estudios Mexicanos y Centroamericanos, 1997.

Calvo, Thomas. "Ordenanzas municipales para el buen gobierno." En Horizontes de monarquía. Siete estudios de caso desde el "Potosí" mexicano. Coord. de Óscar Mazín Gómez y Armando Hernández Soubervielle, 51-94. México: El Colegio de San Luis, 2018.

Chevalier, François. La formación de los latifundios en México. Haciendas y sociedad en los siglos XVI, XVII y XVIII. México: Fondo de Cultura Económica, 1999.

Cramaussel, Chantal. "El Camino Real de Tierra Adentro. De México a Santa Fe." En Rutas de la Nueva España. Ed. de Chantal Cramaussel, 299-327. Zamora, Michoacán: El Colegio de Michoacán, 2006.

Dusenberry, William. The Mexican Mesta. The Administration of Ranching in Colonial Mexico. Urbana: University of Illinois Press, 1963.

Enciso Contreras, José. Ordenanzas de Zacatecas del siglo Xvi y otros documentos normativos neogallegos. Serie Elías Amador. Zacatecas: Ediciones del Ayuntamiento de Zacatecas; Zacatecas: Universidad Autónoma de Zacatecas, Facultad de Derecho, 1998.

Flores Olague, Jesús, Mercedes de Vega, et al. Breve historia de Zacatecas. México: El Colegio de México; México: Fondo de Cultura Económica, 1996. 
González Jácome, Alba. "Breve historia de los huertos en México." Ponencia presentada en el Coloquio "México y sus historias: miradas múltiples", México, 29 de agosto de 2012, Universidad Iberoamericana.

Hernández Soubervielle, José Armando. De piedra y maíz. Las alhóndigas y el abastecimiento de granos en San Luis Potosí durante el virreinato. México: El Colegio de San Luis, 2013.

Hillerkuss, Thomas. "Una sociedad en construcción. La organización de la élite minera en Zacatecas durante el siglo xvi." En México a la luz de sus revoluciones, coord. de Laura Rojas y Susan Deeds, 63-101. México: El Colegio de México, 2017.

Lacueva Muñoz, Jaime J. La plata del rey y sus vasallos. Minería y metalurgia en México (siglos XVI y XVII). Sevilla: Consejo Superior de Investigaciones Científicas, Escuela de Estudios Hispano-Americanos; Sevilla: Universidad de Sevilla; Sevilla: Diputación de Sevilla, 2010.

Lacueva Muñoz, Jaime J. “Zacatecas: norte imperial." En Historia del Reino de la Nueva Galicia. Coord. de Thomas Calvo y Aristarco Regalado Pinedo. Guadalajara: Universidad de Guadalajara, 2016.

Lira, Andrés, "Los bosques en el virreinato (Apuntes sobre la visión política de un problema)." Relaciones. Estudios de Historia y Sociedad, v. 11, n. 41 (1990): 117-127.

Machuca Chávez, Paulina. "Alcohol, mineros y operarios en la Nueva España (siglos XVI-XVIII).” En Alcohol y trabajo en América Latina. Siglos XVII-XX. Coord. de Patricio Herrera González y Juan Carlos Yáñez Andrade, 17-41. Valparaíso: América en Movimiento Ediciones; Valparaíso: Universidad de Valparaíso, Centro de Investigación en Innovación, Desarrollo Económico y Políticas Sociales, 2019.

Machuca Chávez, Paulina. El vino de cocos en la Nueva España. Historia de una transculturación en el siglo XVII. Zamora, Michoacán: El Colegio de Michoacán, 2018.

Martín Ornelas, José Manuel. "La organización económica regional y el abasto urbano. El trigo y el maíz en Zacatecas. 1749-1821.” Tesis para obtener el grado de doctor en Humanidades, Universidad Autónoma de Zacatecas, Zacatecas, 2008.

Matesanz, José. "Introducción a la ganadería en Nueva España, 1521-1535." Historia Mexicana, v. 14, n. 4 (1965): 533-566.

Matilla Tascón, Antonio. Abastecimiento de carne a Madrid, 1477-1678. Madrid: Instituto de Estudios Madrileños, 1994.

Mota y Escobar, D. Alonso de la. Descripción geográfica de los reinos de Nueva Galicia, Nueva Vizcaya y Nuevo León por D. Alonso de la Mota y Escobar. Introd. de Joaquín Ramírez Cabañas, 2a. ed. México: Pedro Robredo, 1940. 
Studnicki-Gizbert, Daviken y David Schecter. "The Environmental Dynamics of a Colonial Fuel-Rush: Silver Mining and Deforestation of New Spain, 1522 to 1810." Environmental History, v. 15, n. 1 (Oxford University Press on behalf of Forest History and American Society for Environmental History, 2010). https://doi.org/10.1093/envhis/emq007.

Quiroz, Enriqueta. Entre el lujo y la subsistencia: mercado, abastecimiento y precios de la carne en la ciudad de México, 1750-1812. México: El Colegio de México; México: Instituto de Investigaciones Dr. José María Luis Mora, 2005.

Vázquez de Warman, Irene. "El pósito y la alhóndiga en la Nueva España.” Historia Mexicana, v. 17, n. 3 (1968): 395-426. 